\begin{abstract}
BaCkground: Psoriatic plaques have been shown to contain increased levels of proinflammatory cytokines. Serum levels of interleukin (IL)-6, IL-7, IL-8, and interferon (IFN)- $\gamma$ have been reported elevated in psoriatic patients.

Aim: To evaluate serum cytokine profiles in psoriasis patients by improved enzyme-linked immunosorbent assay (ELISA) technique and to correlate these levels with disease severity.

Methods: We analyzed single serum samples from 10 patients with active untreated psoriasis, two patients with active treated psoriasis, and five healthy volunteers for major $T$ helper type 1 and $T$ helper type 2 cytokines using the LINCOplex ELISA multi-analyte detection system that permits simultaneous detection of multiple cytokines from a single sample. The disease severity, including erythema, induration, scale, and surface area, was assessed.

Results: IFN- $\gamma$ was markedly elevated in all sera from psoriasis patients, $33.8 \pm 1.3 \mathrm{pg} / \mathrm{ml}$ (mean \pm standard error) versus $8 \pm 1.5 \mathrm{pg} / \mathrm{ml}$ for normal controls $(p<$ 0.01 ), and positively correlated with all indices of disease severity (Spearman $r>0.6$ ). IL-8 was also increased in psoriasis patients $(24.4 \pm 1.8 \mathrm{pg} / \mathrm{ml})$ versus normal controls $(3.6 \pm 1.2 \mathrm{pg} / \mathrm{ml})(p<0.05)$ and positively correlated with the degree of erythema (Spearman $r>0.6$ ). Mean IL-12 levels were decreased in sera from psoriasis patients $(8.5 \pm 1.2 \mathrm{pg} / \mathrm{ml})$ compared with normal controls $(42.2 \pm 5.3 \mathrm{pg} / \mathrm{ml})$ $(p<0.01)$. Also, serum IL-10 levels were below detection levels in psoriatics compared with controls $(6.4 \pm 1.3 \mathrm{pg} / \mathrm{ml})$.

Conclusions: This new ELISA system allowed rapid and reliable detection of numerous cytokines in single serum samples from patients with psoriasis. We observed that IFN- $\gamma$ and IL-8 cytokines were elevated in psoriatics and correlated with parameters of disease severity while IL-10 and IL-12 were decreased.
\end{abstract}

\section{Simultaneous measurement of multiple Th1 and Th2 serum cytokines in psoriasis and correlation with disease severity}

\author{
Sharon E. Jacob ${ }^{1}$, Mehdi Nassiri ${ }^{2}$, Francisco A. \\ Kerdel $^{1}$ and Vladimir Vincek ${ }^{2, C A}$
}

${ }^{1}$ Department of Dermatology and Cutaneous Surgery and ${ }^{2}$ Department of Pathology, University of Miami/ Jackson Memorial Medical Center, Holtz Center, Room 2042, 1611 NW 12 Avenue, Miami, FL 33136, USA

\footnotetext{
${ }^{\mathrm{CA}}$ Corresponding author

Tel: + 13055856484

Fax: + 13053269523

E-mail: vvincek@med.miami.edu
}

\section{Introduction}

Psoriasis vulgaris is a persistent chronic relapsing, inflammatory and hyperproliferative skin disorder with genetic predisposition. In the United States, psoriasis affects approximately $2 \%$ of the population. There is currently no good experimental model for psoriasis. Psoriatic plaques have been shown to contain increased levels of cytokines, including tumor necrosis factor-alpha (TNF- $\alpha)$, interleukin (IL)-6, IL- $1 \alpha$, IL-1r $\alpha$, IL-8R ${ }^{1,2}$ and macrophage migration inhibitory factor, ${ }^{3,4}$ whereas IL-10 expression has been shown to be lower than in perilesional skin. ${ }^{5}$

While TNF- $\alpha$ appears to be playing a critical role in propagation of lesional psoriatic plaques, serum TNF$\alpha$ has been reported to be within normal limits. ${ }^{6}$ On the contrary, serum IL-6, IL-8, and interferon-gamma (IFN- $\gamma$ ) have been reported significantly elevated in the sera of psoriatic patients with active disease. ${ }^{5,7}$ It has been reported that IL-6 elevation positively correlates with Psoriatic Assessment and Severity Index scores. A negative correlation has been seen with IL- $1 \alpha$ levels and disease activity. ${ }^{6,7}$ In patients with psoriatic arthritis sIL-2R, IL-1r $\alpha$, and IL-10 were found to be elevated in the sera during periods of disease activity. Of special note, sIL-2R has been shown to have a significant association with the severity of skin involvement, whereas IL-1r $\alpha$ correlates with the number of tender/swollen joints. ${ }^{5}$ The current state of knowledge suggests that over-expression of proinflammatory, type 1 , cytokines and the under-expression of type 2 cytokines are of pathological significance in psoriasis. As the psoriatic story unfolds, we need more reliable and rapid tests to elucidate the biological relevance of Th1 versus Th2 cytokines in the pathogenesis of psoriasis. Moreover, the measurement of these cytokines may allow for better monitoring and prediction of disease activity. 


\section{Methods}

Twelve psoriatic patients were admitted to dermatology inpatient ward at Cedars Medical Center/University of Miami for Goeckerman therapy for recalcitrant psoriasis. $^{8}$ Of the 12 patients selected for enrollment in this University of Miami Institutional Review Board-approved study, nine had plaque psoriasis, two had guttate and one had erythroderma. Out of nine plaque psoriasis patients, seven had received no systemic therapy and two were on methotrexate. The guttate and erythrodermic patients had not received systemic therapy at the time of the inclusion in the study.

At the time of admission, the patients were assessed by the same physician and assigned scores for four indices of disease severity. Independent scores for body surface area (BSA) involvement, degree of erythema, thickness and scale of a target plaque that denoted the severity of the patients disease state were given. Score for percent BSA involvement was from $1(0-20 \%), 2(20-40 \%), 3$ (40-60\%), $4(60-80 \%)$ to $5(80-100 \%)$. A target lesion was selected for assessment; thickness was scored from 0 (flat), 1 (minimal thickness), 2 (mild), 3 (moderate) to 4 (severe). The target lesion was assessed for erythema as follows: 0 (none), 1 (minimal), 2 (mild), 3 (moderate), and 4 (severe). Scaling of the target lesion was assessed as: 0 (none), 1 (minimal), 2 (mild), 3 (moderate), 4 (severe). On admission the patient's vital signs, weight and baseline laboratory data were drawn. Each patient consented to the use of residual blood from a routine laboratory draw to measure serum cytokine levels. In addition five healthy non-psoriatic volunteers with no family history of psoriasis consented to have their blood drawn for the purpose of measuring serum cytokine levels.

For the purpose of the assay, $25 \mu \mathrm{l}$ of sera was used to measure IL-1 $\beta$, IL-2, IL-4, IL-5, IL-6, IL-8, IL-10, IL12 , IFN- $\gamma$, granulocyte-monocyte colony-stimulating factor (GM-CSF), and TNF- $\alpha$. Measurements were made by LINCOplex simultaneous multi-analyte detection system (Linco Research Inc., St Charles, Missouri, USA) following manufacturer's instruction. All cytokines are simultaneously measured from a single specimen. The LINCOplex assay was shown to produce results comparable with standard single cytokine assays. ${ }^{9}$ Statistical analysis of the results was performed with the aid of Statistical Software (StatSoft, Tulsa, Oklahoma, USA).

\section{Results}

Serum cytokine levels of major Th1 and Th2 type cytokines were simultaneously measured from the single specimen prior to initiation of therapy in patients with recalcitrant psoriasis. Results are presented in Table 1. Th2 type cytokine, IL-10, was undetectable in psoriatic patients, of any type, compared with controls $(6.4 \pm 1.3 \mathrm{pg} / \mathrm{ml})$. The IL-5, another of the Th2 type cytokines, was not detected in normal nor psoriatic patients. The remaining two tested Th2 type cytokines, IL- 4 and IL-6, were present at similar low level in psoriasis patients and normal controls, with the exception that in two psoriasis patients both were significantly increased.

When compared with normal sera, the level of Th1 type cytokine IFN- $\gamma$ was markedly elevated in all sera from psoriasis patients, $33.8 \pm 1.3 \mathrm{pg} / \mathrm{ml}$ (mean \pm standard error) versus $7.6 \pm 2.2 \mathrm{pg} / \mathrm{ml}$ for normal controls $(p<0.01)$. Besides, when IFN- $\gamma$ was compared with clinical indices of disease severity, it showed positive correlation (Spearman rank order test: BSA, $r=0.64$; thickness, $r=0.62$; erythema, $r=$ 0.7 ; scale, $r=0.67 ; p<0.05)$. The IL-2, another Th-1 type cytokine, did not show elevation in the serum of psoriasis patients, whereas IL-12 was decreased in psoriatics $(8.5 \pm 1.2 \mathrm{pg} / \mathrm{ml})$ compared with normal controls $(42.2 \pm 5.3 \mathrm{pg} / \mathrm{ml}, p<0.01)$. From three tested cytokines that cannot be clearly defined as Th1 or Th2, IL-8, TNF- $\beta$, and GM-CSF, only IL-8 was significantly increased in serum of psoriatic patients $(24.4 \pm 1.8 \mathrm{pg} / \mathrm{ml})$, compared with normal controls $(3.6 \pm 1.2 \mathrm{pg} / \mathrm{ml}, p<0.05)$ and positively correlated with the degree of erythema (Spearman $r>0.6 ; p<$ 0.05). Fig. 1 illustrates the correlation of the erythema score with serum level of IFN- $\gamma$ and IL-8.

\section{Discussion}

Our study shows for the first time that 11 major human cytokines, including the major Th1 and Th2 cytokines, were simultaneously quantitatively determined from a single sample in the serum of psoriatic patients. In prior studies solitary cytokines were measured in separate ELISA studies that can potentially cause variation in results due to the variation between samples and individual experiments. ${ }^{7,10}$ In our testing using LINCOplex kit and Luminex technology (Linco Research Inc.) 11 captured antibodies for specific cytokines were coupled to specific bead sets with different fluorophores. After analytes were bound to the capture antibody on the beads, a detector antibody coupled to phycoerythrin is used as a reporter. The assay was read using a dual laser system. Using multiple bead sets, 11 different assays were simultaneously performed and detected on the individual serum samples. Performing cytokine testing that way means variation between test samples and individual experiments is eliminated. In addition, the cost of testing is significantly decreased. 


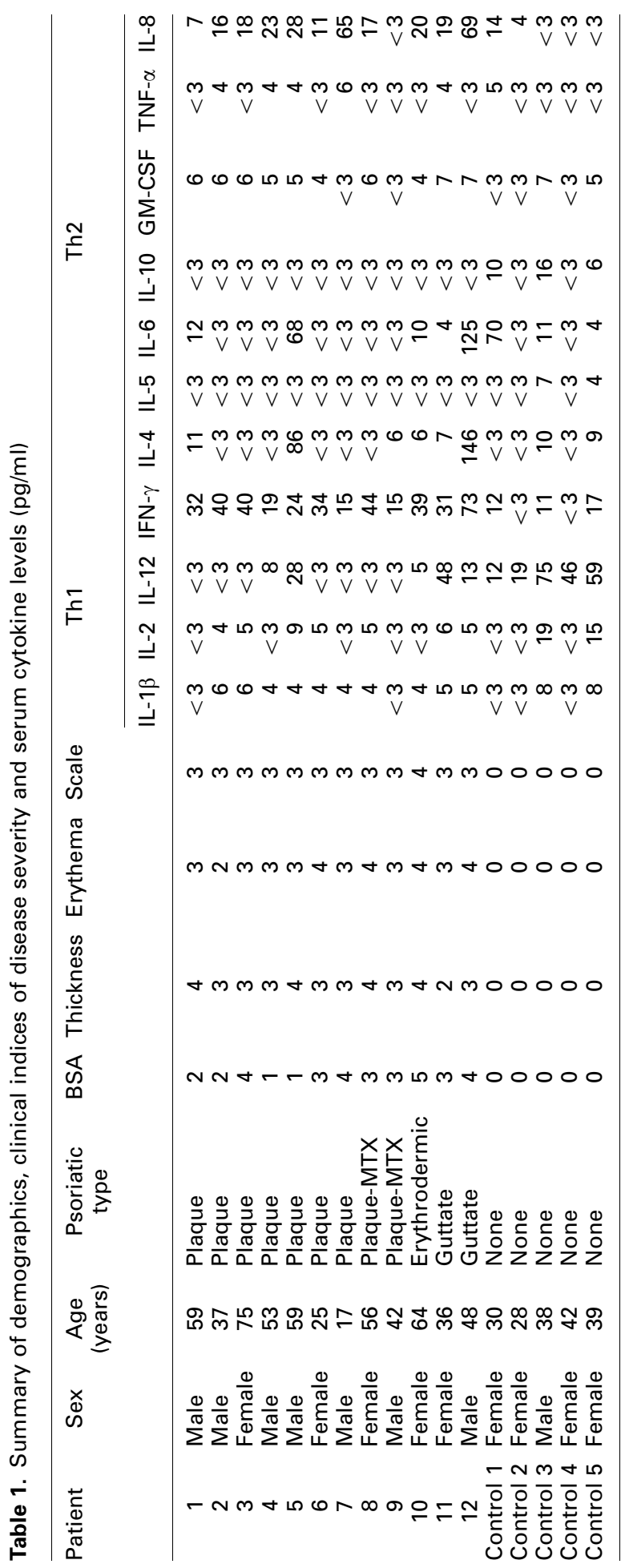

Over-expression of proinflammatory, type 1, cytokines is believed to be of pathogenic significance in psoriasis. ${ }^{11}$ Our data confirm previously published data that the Th1 type cytokine IFN- $\gamma$ is elevated in the serum of psoriatic patients. In addition our data suggest that the serum level of IFN- $\gamma$ correlates with clinical indices of severity that could potentially be used to monitor progression of traditional psoriatic therapy. However, the other two tested Th1 type cytokines, IL-2 and IL-12, did not show significant increase between psoriatic patients and normal controls. On the contrary, IL-12 showed a significant decrease in psoriatic patients. A normal level of IL-2 could be expected since it induces proliferation of activated $\mathrm{T}$ and $\mathrm{B}$ cells. On the contrary, the fact that IL-12 was not elevated but rather decreased was not expected, as IL-12 is a fundamental cytokine for Th1 differentiation. It induces proliferation and IFN- $\gamma$ production by Th1 lymphocytes. The observation that IFN- $\gamma$ is elevated in psoriatic patients, while IL-12 is decreased, suggests that in psoriasis patients IFN- $\gamma$ production might be induced by another pathway.

Among the tested Th2 type cytokines, we found a significant decrease of the IL-10 serum cytokine level in the patients with psoriasis. This dramatic suppression of IL-10 was a constant feature. IL-10 is an antiinflammatory cytokine that suppresses macrophage production of cytokines/chemokines and enhances soluble cytokine receptor release. It is known that IL10 modulates antigen presentation by dendritic cells and suppresses co-stimulatory reactions by a direct action on T cells. McInnes et al. demonstrated that a 28-day course of subcutaneous recombinant human IL-10 provided clinically significant improvement of the skin but not the articular disease in 29 patients with psoriatic arthritis. The administration of recombinant human IL-10 also caused a selective functional suppression of the pro-inflammatory activities of circulating monocytes. ${ }^{12}$ Low levels of IL-10 have been observed in the cutaneous lesions of psoriatic patients and with established antipsoriatic therapy higher IL-10 mRNA levels can be found in peripheral blood mononuclear cells. Thus, it is consistent that serum levels of IL-10 in our patients were decreased. Interestingly, patients with psoriatic arthritis have been shown to have increased serum levels of IL$10,{ }^{1}$ which probably represents a compensatory attempt of these patients with both skin and articular disease to regulate the inflammatory process. Other tested Th2 type cytokines, IL-4, IL-5, and IL-6, had serum levels comparable with normal controls. Although previously IL-6 was shown to be elevated in serum of patients with active psoriasis, ${ }^{1}$ our data did not confirm this. However, there were two patients of the 12 that demonstrated elevated serum IL-4 and IL-6 levels, in addition to one normal control that had elevated IL-6. Elevated IL-4 and IL-6 did not 


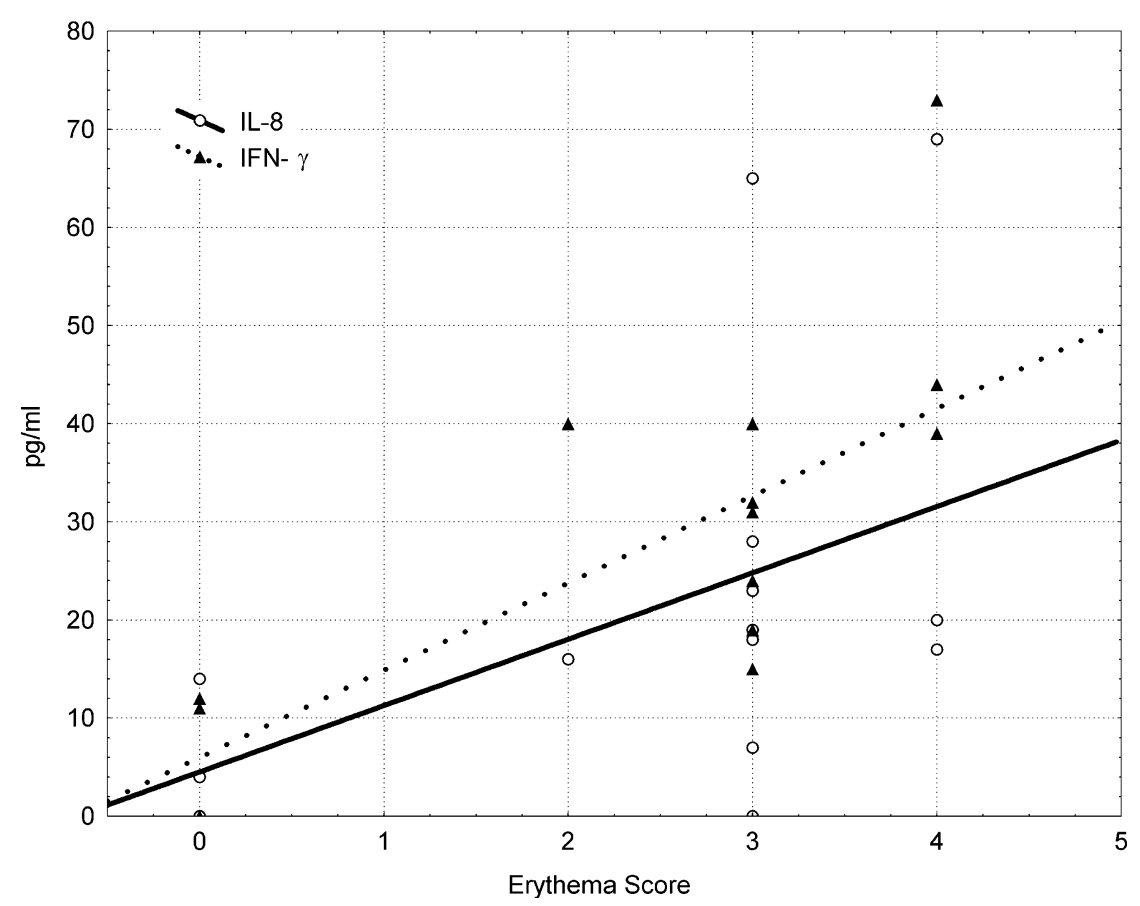

FIG. 1. Scatter plot of IFN- $\gamma$ (closed triangles) and IL-8 (open circles) serum levels in correlation with erythema score. Lines denote the regression curve fit.

correlate with BSA score. We do not have a good explanation for that observation.

As per previous reports, we found that patients with psoriatic skin disease had normal serum levels of TNF- $\alpha$, significantly higher IFN- $\gamma$ and moderately increased IL-8. ${ }^{4,6,7}$ We were able to furthermore demonstrate an association between serum level of IFN- $\gamma$ and clinical indices of severity.

Among the tested cytokines there were three (TNF$\alpha$, GM-CSF, IL-8) that cannot be clearly classified as Th1 or Th2. TNF- $\alpha$, while significantly elevated in the lesional psoriatic plaques of patients, is known to be at normal levels in the psoriatic patient's sera. Our data support previous findings. Even in the patients with an erythroderma the TNF- $\alpha$ level was normal. Of note, TNF- $\alpha$ levels have been noted to remain unchanged after standard psoriasis therapy. ${ }^{7}$ GMCSF was not elevated in our patient population. To our knowledge GM-CSF in psoriasis has not been previously reported.

We found IL- 8 to be elevated in our patients. The level of IL-8 was found to positively correlate with degree of erythema. IL-8 is a known chemotactic factor for neutrophils and, by an indirect mechanism, induces neutrophil degranulation. As neutrophils are present in the epidermis of the psoriatic lesions, it is possible that IL-8 aids in their recruitment and thus contributes to the erythema. While the kinetics of IL-8 in the serum of patients with psoriasis are not known, one could presume that IL- 8 could be a good index for the inflammatory process and a crucial element in the cellular activation of psoriasis.
Among the tested psoriatic patients, two were on methotrexate therapy. This number of patients is not adequate to draw definitive conclusive data.

We realize the limitations of our data, since we had a small number of patients. Yet, with the mounting body of scientific evidence in the literature, and our congruent results we are able to conclude that psoriasis is not, as many times inferred, a Th1 versus Th2 disease. Rather it is a complex disease in which the cytokine network is dysregulated with only some components of the Th1-Th2 cytokine network being involved. The new technique that we utilized in our study is rapid, flexible, and inexpensive compared with the traditional ELISA, and requires a very small amount of serum for testing. It could be modified in the future, to test specific cytokines relevant in psoriasis, to better understand the pathophysiology of psoriasis, and to possibly serve as an objective tool to assess severity, progression and prognosis. Furthermore, the manipulation of these cytokines may allow for new and more specific therapeutic alternatives.

ACKNOWLEDGEMENTS. The authors thank Jennean Knowles, Gloria Jimenez MD, Joseph Soó MD, and Clay L. Brown for excellent technical support.

\section{References}

1. Elkayam O, Yaron I, Shirazi I, Yaron M, Caspi D. Serum levels of IL-10, IL-6, IL-1R and sIL-2R in patients with psoriatic arthritis. Rheumatol Int 2000; 19: $101-105$.

2. Elder JT. Cytokine and genetic regulation of psoriasis. Adv Dermatol 1995; 10: 99-133. 
3. Shimizu T, Nishihira J, Mizue Y, et al. Histochemical analysis of macrophage migration inhibitory factor in psoriasis vulgaris. Histochem Cell Biol 2002; 118: 251-257.

4. Shimizu T, Nishihira J, Mizue Y, et al. High macrophage migration inhibitory factor (MIF) serum levels associated with extended psoriasis. $J$ Invest Dermatol 2001; 116: 989-990.

5. Pietrzak A, Koziol-Montewka M, Lecewicz-Torun B, Krasowska D. Is there any correlation between the total number of neutrophils in plasma and concentration of interleukin- 8 in psoriatic patients? Med Sci Monit 2000; 6: $867-870$

6. Gomi T, Shiohara T, Munakata T, Imanishi K, Nagashima M. Interleukin 1 alpha, tumor necrosis factor alpha, and interferon gamma in psoriasis. Arch Dermatol 1991; 127: 827-830.

7. Szepietowski JC, Bielicka E, Nockowski P, Noworolska A, Wasik F. Increased interleukin-7 levels in the sera of psoriatic patients: lack of correlations with interleukin-6 levels and disease intensity. Clin Exp Dermatol 2000; 25: 643-647.
8. Le Vine MJ, White HA, Parrish JA. Components of the Goeckerman regimen. J Invest Dermatol 1979; 73: 170-173.

9. Hildesheim A, Ryan RL, Rinehart E, Nayak S, Wallac D, Castle PE, Niwa S Kopp W. Simultaneous measurement of several cytokines using small volumes of biospecimens. Cancer Epidemiol Biomarkers Prev 2002; 11 $1477-1484$

10. el Barnawi NY, Giasuddin AS, Ziu MM, Singh M. Serum cytokine levels in psoriasis vulgaris. Br J Biomed Sci 2001; 58: 40-44

11. Cotran RS, Kumar V, Collins T. Robbins Pathologic Basis of Disease, 6th edition, Philadelphia: W.B. Saunders Co., 1999: 1199.

12. McInnes IB, Illei GG, Dannin CL, et al. IL-10 improves skin disease and modulates endothelial activation and leukocyte effector function in patients with psoriatic arthritis. J Immunol 2001; 167: 4075-4082.

\section{Received 8 August 2003 \\ Accepted 25 August 2003}




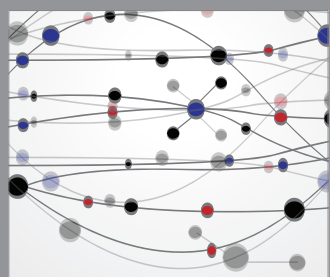

The Scientific World Journal
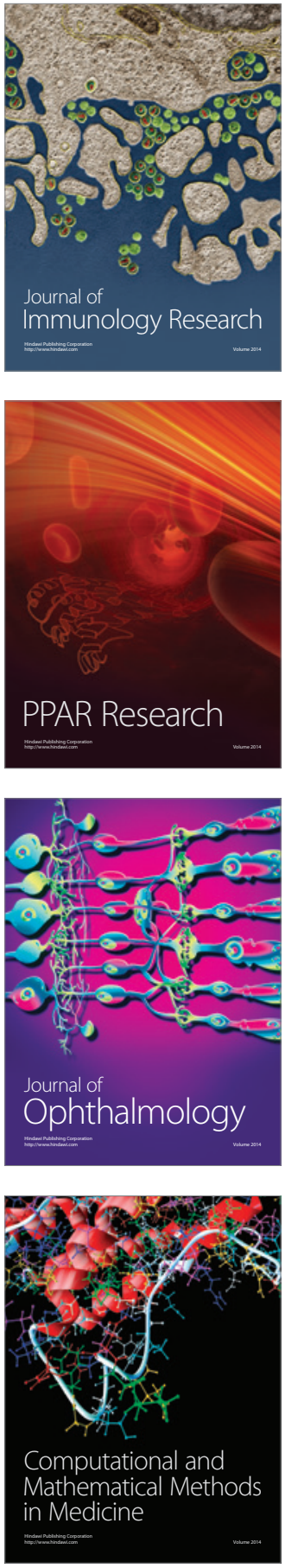

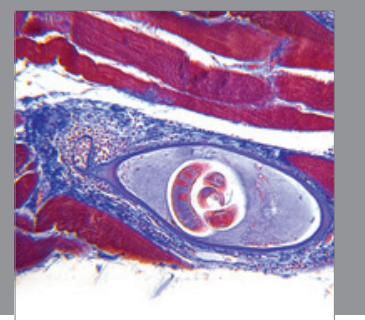

Gastroenterology

Research and Practice
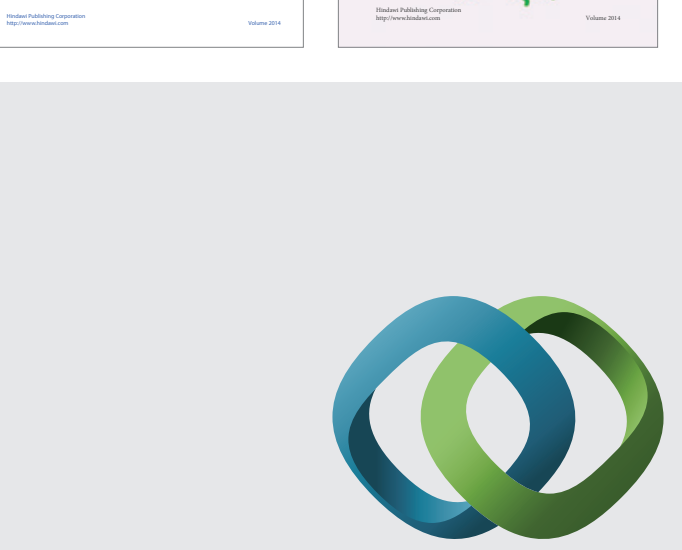

\section{Hindawi}

Submit your manuscripts at

http://www.hindawi.com
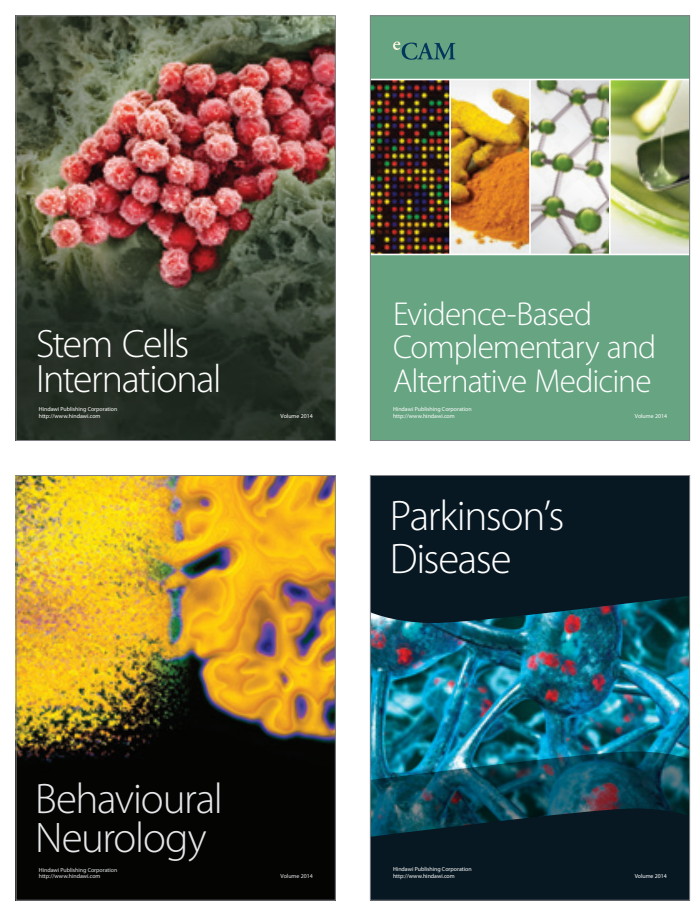

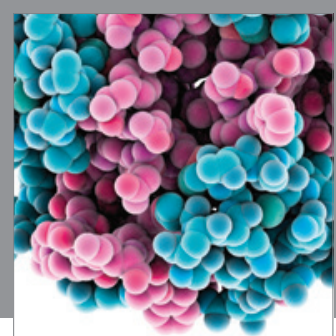

Journal of
Diabetes Research

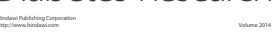

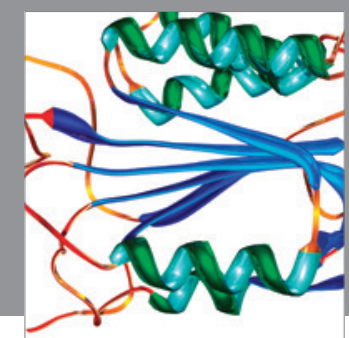

Disease Markers
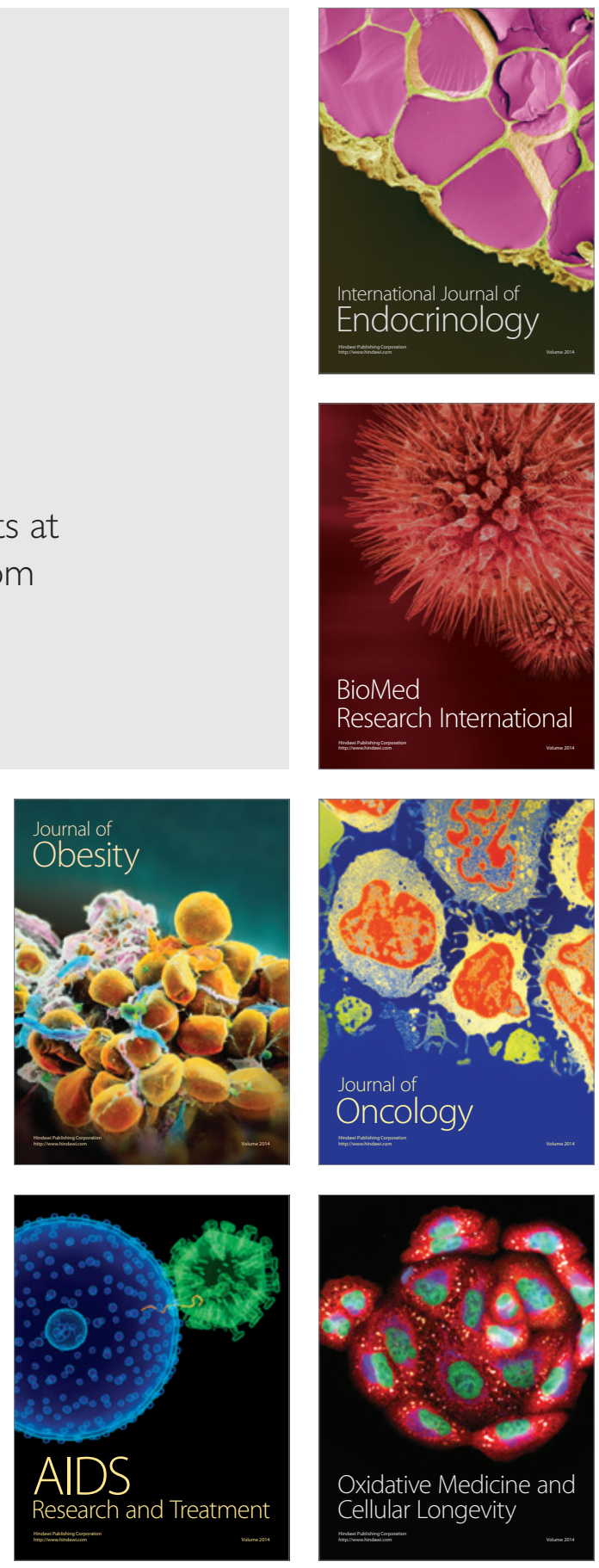